\title{
2021. Un año de elecciones y retos en Chile
}

\author{
Jesús Castellanos Vásquez*
}

https://doi.org/10.35242/RDE_2022_22_33_13

Nota del Consejo Editorial

Recepción: 15 de noviembre de 2021.

Revisión, corrección y aprobación: 14 de enero de 2022.

Resumen: El 2021 fue un año de cambios y de retos en Chile, producto de un nuevo marco político gestado, entre otras razones, por las protestas de 2019. Conforme al Plebiscito Nacional de 2020 y por motivos de la pandemia, flagelo que ha ocasionado varias postergaciones electorales, se definió un calendario contentivo de todos los cargos de elección popular, a saber: elecciones subnacionales (gobernadores regionales, alcaldes y concejales), junto con la Convención Constitucional, así como la segunda vuelta para los gobernadores regionales, primarias presidenciales oficiales, "primarias presidenciales convencionales" (no organizadas por el Servicio Electoral, máximo órgano electoral administrativo) y, para cerrar, elecciones nacionales: presidente de la República, renovación parcial de senadores, diputados al Congreso y consejeros regionales, con una eventual segunda vuelta presidencial. No queda duda, en virtud de los eventos realizados, de la fortaleza institucional electoral y de la cultura política en Chile; no obstante, son varias las materias que pudieran evaluarse, tales como: actualización del registro electoral, financiamiento electoral, reelección, regulación de encuestas electorales, y políticas de acción afirmativas por género y otros grupos. Una mayor autonomía del ente administrativo electoral y la necesidad de formalizar la observación electoral pudieran ser claves para esta revisión.

Palabras clave: Situación política / Situación social / Protestas / Organización electoral / Administración electoral / Proceso electoral / Chile.

Abstract: 2021 is a year of changes and challenges in Chile amid a new political context caused, among other reasons, by the 2019 protests. According to the 2020 National Plebiscite, and due to the pandemic, scourge that has led to several electoral delays, a calendar containing all popular election posts was set as follows: subnational elections (Regional Governors, official presidential primaries, "conventional presidential primaries" (not organized by the Electoral Service, top electoral management body), and to wrap up, national elections: President of the Republic, partial renewal of Senators. Members to Congress, and Regional Councilors with a possible presidential runoff. There is no doubt, considering the events that took place, about the electoral institutional strength and political culture of Chile. Yet, there are several subjects that can be assessed such as: update of electoral roster, electoral funding, reelection, regulation of electoral polls, and policies of affirmative action per gender and other groups. A greater autonomy of the electoral body and the need to formalize electoral observation could be key to this assessment.

Key Words: Political situation / Social situation / Protests / Electoral organization / Electoral management / Electoral process / Chile.

\footnotetext{
* Venezolano politólogo, correo jacv1970@gmail.com. Licenciado en Ciencias Políticas y Administrativas de la Universidad Central de Venezuela (1992), especialista en Comunicación Organizacional de la Universidad Católica Andrés Bello (2001) y magíster en Gestión y Políticas Públicas de la Universidad de Chile (1998). Actualmente, aspirante al Doctorado en Ciencias Políticas. Funcionario de carrera del Consejo Supremo Electoral/Consejo Nacional Electoral de Venezuela (1992-2016). Profesor de pregrado UCAB (1999-2001), de pregrado UCV (1994-1996) y del postgrado de Procesos y Sistemas Electorales UCV (1999-2015). Ha sido consultor de IDEA Internacional, Transparencia Electoral (Argentina) y de varias organizaciones venezolanas. Colaborador del Proyecto de Integridad Electoral (The Electoral Integrity Project) en 2017 y 2018 y en la Encuesta Mundial de Partidos Políticos 2019 (Global Party Survey) e Integrante de ACE Project.
} 


\section{DERECHO EIECTORAL}

\section{INTRODUCCIÓN}

Chile enfrenta hoy un enorme desafío histórico. Tras las fuertes protestas iniciadas en octubre de 2019, surgió la urgencia entre los principales actores del sistema político de buscar soluciones, trayendo como resultados la celebración del Plebiscito Nacional 20201, consulta destinada a aprobar 0 , por el contrario, rechazar la posibilidad de modificar la actual Constitución de la República de Chile -adoptada bajo el régimen de Augusto Pinochet en 1980 aunque a la fecha posee numerosos cambios-, así como la definición del tipo de órgano constituyente, cuerpo responsable de elaborar la propuesta de la nueva carta magna y cuyos miembros fueron escogidos en su totalidad por mandato del citado plebiscito ${ }^{2}$, a través del voto popular en una consulta específica posterior ${ }^{3}$. Todo ello, gracias al Acuerdo por la Paz Social y la Nueva Constitución del 15 de noviembre de 2019 y a la Ley n. ${ }^{\circ} 21.200$.

Pese a la calificación como sistema político institucional estable que ostenta Chile desde hace muchos años y que se refleja en índices de la más variada gama como The Electoral Integrity Project, The Economist o Freedom House, en lo que corresponde a la materia político-electoral o The World Justice Project sobre el respeto al Estado de derecho, no es menos cierto que existe un alto y sostenido nivel de insatisfacción con relación a la democracia en la última década (Corporación Latinobarómetro, 2021), aunque el apoyo como régimen político factible $^{4}$ sigue siendo alto e incluso creció en el año 2020:

Hoy Chile goza de uno de los más altos niveles de apoyo a su democracia desde que se comienza a medir hace un cuarto de siglo en $1995 . .$. El apoyo a la democracia no está relacionado con la confianza en las instituciones de la democracia y no se ve afectado por ello.

\footnotetext{
${ }^{1}$ Sobre el Plebiscito Nacional 2020, ver https://www.plebiscitonacional2020.cl/

2 Para más detalles de los resultados de Plebiscito Nacional 2020, ver https://www.servel.cl/resultadosprovisorios-plebiscito-nacional-2020/.

3 Sobre la Elección de la Convención Constitucional 2021, ver https://elecciones2021.servel.cl/convencionales-constituyentes/

${ }^{4}$ La ponderación que se hace de factible es en atención a lo que señala el estudio citado como democracia churchiliana: "La democracia es el mejor sistema de gobierno excepto por todos los otros" (Latinobarómetro, 2020, p. 13). Para una revisión más completa del estado actual de la democracia en Chile, recomendamos la lectura de Informe Chile 2020 de la Corporación Latinobarómetro.
} 


\section{DERECHO ELECTORAL}

La democracia que tenemos no es la que quieren los chilenos. Casi ningún chileno dice que lo que tenemos es una democracia plena ( $2 \%)$, aumenta once puntos porcentuales de $43 \%$ en 2018 a $53 \%$ en 2020 los que dicen que nuestra democracia tiene grandes problemas, y aumenta de $6 \%$ a 15 $\%$ en el mismo período los que dicen que esto no es una democracia. No hay duda alguna respecto de la dureza de la crítica a nuestra manera de hacer democracia. (Latinobarómetro, 2021, p. 12).

Este nivel de insatisfacción no solo se registra a través de las percepciones sobre el funcionamiento de la democracia, sino también, de acuerdo a informes como el arriba citado y a politólogos como Pamela Figueroa (2021), en el cuestionamiento a instituciones del Estado y del sistema político, tales como el poder ejecutivo, congreso y partidos políticos, entre otros. Estas percepciones podrían formar parte del conjunto de variables explicativas de la significativa abstención electoral en los últimos procesos electorales:

Si bien Chile ha sido considerado una de las democracias más estables en América Latina, desde la década de 2010 se vino forjando una crisis de confianza y legitimidad en las instituciones políticas, que se expresó en bajos indicadores de apoyo a los partidos políticos, el Congreso, el Gobierno, y las principales instituciones públicas del país, así como también una baja sostenida en la participación electoral. (Figueroa, 2021, s. p).

En este marco inicia el año 2021, en el que se plantea no solo la elección del presidente de la República, renovación parcial de senadores, diputados al Congreso y consejeros regionales ${ }^{5}$, sino que formaban parte del calendario electoral, por razones de pandemia (Chile, 2019b), las elecciones de alcaldes, concejales, gobernadores regionales, figuras que serían electas por primera vez en el país (Chile, 2018), además del cuerpo constituyente, denominado Convención Constitucional. Dado el ordenamiento jurídico vigente, a ese cronograma de elecciones se suman las primarias para presidente de la

\footnotetext{
${ }^{5}$ Los consejeros regionales se erigirán por última vez en las Elecciones Nacionales de 2021. A partir de 2024, la elección se realizará en conjunto con los gobernadores regionales e instancias municipales. Ley 21.073. https://www.bcn.cl/leychile/navegar?idNorma $=1115503$
} 
República, las de segunda vuelta para gobernadores regionales cuando fuese necesario ${ }^{6}$ y la eventual segunda vuelta de la elección presidencial ${ }^{7}$, a lo que se debe agregar la consulta presidencial realizada por un grupo de partidos políticos, calificada como primaria convencional $^{8}$. El COVID-19, al igual que con el Plebiscito Nacional de 2020, ocasionó postergaciones en las fechas de las elecciones de la Convención Constitucional, alcaldes, concejales y gobernadores regionales, así como la correspondiente a la segunda vuelta de este último cargo y las primarias presidenciales (Chile, 2021). La tabla 1 presenta el calendario de los procesos electorales en Chile para 2021.

\section{Tabla 1}

\section{Calendario electoral. Chile 2021}

\begin{tabular}{|c|c|c|}
\hline Tipo de elección & Cargos por elegir & Fecha definitiva \\
\hline $\begin{array}{l}\text { Convención Constitucional y General, } \\
\text { (regional) (realizado) }\end{array}$ & $\begin{array}{l}\text { Constituyentes, gobernadores } \\
\text { regionales, alcaldes y } \\
\text { concejales }\end{array}$ & 15 y 16 de mayo de 2021 \\
\hline $\begin{array}{lcc}\text { Segunda } & \text { vuelta } & \text { gobernadores } \\
\text { regionales (realizado) } & \\
\end{array}$ & Gobernadores regionales & 13 de junio de 2021 \\
\hline Primarias presidenciales (realizado) & & 18 de julio de 2021 \\
\hline $\begin{array}{l}\text { "Primarias presidenciales } \\
\text { convencionales" (realizado) }\end{array}$ & & 21 de agosto de 2021 \\
\hline Generales nacionales & $\begin{array}{l}\text { Presidente de la República, } \\
\text { senadores (parcial), diputados } \\
\text { al Congreso y consejeros } \\
\text { regionales }\end{array}$ & 21 de noviembre de 2021 \\
\hline Segunda vuelta & Presidente de la República & 19 de diciembre de 2021 \\
\hline
\end{tabular}

El objeto del presente artículo es identificar los principales elementos técnicos asociados a los procesos electorales celebrados en Chile

\footnotetext{
${ }^{6}$ El sistema electoral para gobernadores regionales es de mayoría en la que se requiere que el candidato ganador obtenga al menos el $40 \%$ de los votos válidos emitidos. En caso de que ninguno logre ese porcentaje, se deberá ir a una segunda vuelta entre los dos más votados (Ley 21.073). El sistema electoral para alcaldes, por el contrario, es de mayoría simple (Ley 18.695) https://www.bcn.cl/leychile/navegar?idNorma=251693.

7 El sistema electoral para presidente de la República es de mayoría absoluta, es decir, se requiere al menos el $50 \%$ de los votos válidamente emitidos. En caso de no alcanzarse, se convoca a una segunda vuelta el cuarto domingo después de celebrada la primera, entre los dos candidatos más votados (Constitución Política de la República de Chile), https://www.bcn.cl/leychile/navegar?idNorma=242302.

8 El Servicio Electoral (SERVEL) no organizó dicha consulta, de hecho, publicó un comunicado oficial de fecha 16/06/2021, en donde se establece, entre otros puntos, que no existe la figura de las primarias convencionales y que eso "solo induce al error y confunde a los electores". Ver https://www.servel.cl/acuerdo-del-consejo-directivo-sobre-elecciones-primarias-y-consultas-ciudadanaspara-la-determinacion-de-candidatos/
} 


\section{DERECHO EIECTORAL}

durante el año 2021, a la luz de categorías de análisis de sistemas electorales en su sentido amplio, entre otras: registro electoral, cargos por elegir, circunscripciones electorales, sistemas electorales y políticas de acción afirmativa.

\section{Elecciones de la Convención Constitucional, Gobernadores Regionales, Alcaldes y Concejales, 15 y 16 de mayo de 2021}

En el marco de un proceso inédito, el 15 y 16 de mayo, se eligió la Convención Constitucional, así como por primera vez a los gobernadores regionales, junto con los alcaldes y concejales, en unos comicios con un acto de votación de dos días, como parte de las medidas adoptadas para enfrentar el COVID-19. Contó con un padrón de 14900189 electores $^{9}$ y un total de 2768 cargos por elegir distribuidos de la siguiente manera: 155 convencionales constituyentes ${ }^{10}$, 16 gobernadores regionales, 345 alcaldes y 2252 concejales municipales ${ }^{11}$, hecho que podría calificarla como una megaelección, con 16370 candidatos y 744 tipos de boletas ${ }^{12}$ electorales ${ }^{13}$, algunas de las cuales podían alcanzar el tamaño de la página de un diario estándar.

El voto en Chile desde el año 2012 (Ley 20.568) es voluntario ${ }^{14}$. Para el proceso electoral del año 2021 cada votante tenía derecho a ejercer cuatro votos en cuatro cédulas o boletas electorales distintas: una para elegir a un candidato a la Convención Constitucional, una para elegir a un candidato a la Gobernación Regional, una para elegir a un candidato a la Alcaldía Municipal y otra para elegir a un candidato al Concejo Municipal. En todos los casos eran votos uninominales. A tono con el

\footnotetext{
9 Para más información sobre el padrón electoral de las elecciones del 15 y 16 de mayo de 2021, ver https://www.servel.cl/padron-electoral-por-sexo-y-region-elecciones-abril-2021/.

10 Información sobre candidatos postulados a convencionales constitucionales en https://www.servel.cl/wpcontent/uploads/2021/03/BOLETIN_UNICO_CCG_11-03-2021.pdf y pueblos indígenas a la Convención Constitucional https://www.servel.cl/wp-content/uploads/2021/02/BOLETIN_1_CCPI_25-02-2021.pdf.

${ }_{11}$ Para más información sobre requisitos e inhabilidades en la elección de gobernadores regionales, alcaldes y concejales, en 2021, ver https://www.servel.cl/catalogo-de-inhabilidades-e-incompatibilidades-de-cargosde-eleccion-popular/.

${ }^{12}$ Información general y facsímiles de las boletas electorales para las elecciones del 15 y 16 de mayo de 2021 en: https://elecciones2021.servel.cl/informacion-general/.

13 Información sobre el número de candidatos y boletas electorales en: https://elecciones2021.servel.cl/nueva-fecha-para-las-elecciones-15-y-16-de-mayo/.

${ }^{14}$ En la actualidad, se está discutiendo en el Congreso de Chile la posibilidad de retorno al voto obligatorio. Ver https://www.senado.cl/noticias/voto/luz-verde-a-idea-de-legislar-del-proyecto-que-restablece-el-voto, https://www.senado.cl/appsenado/index.php?mo=sesionessala\&ac=detalleVotacion\&votaid=8457.
} 


\section{DERECHO ELECTORAL}

sistema electoral de concejales, definido en la Ley 18.695 y el adoptado para senadores y diputados al Congreso en la Ley 20.840, se decidió que para la elección de la Convención Constitucional se aplicaría un sistema de representación proporcional a través de la fórmula de divisores del Método D'Hondt. El sistema electoral de alcaldes es de mayoría relativa o simple y el de gobernadores regionales es también mayoritario, pero requiere un porcentaje de los votos válidos emitidos $(40 \%)$.

Los principales factores distintivos de este proceso estuvieron referidos a la elección de los convencionales constituyentes, que tuvo como circunscripciones electorales, de acuerdo a lo dispuesto en la Ley 21.200, las mismas definidas para elegir a los diputados al Congreso; es decir, según la Ley 20.840, veintiocho (28) distritos con el correspondiente número de cargos por elegir. Salvo que, y he aquí uno de los rasgos innovadores de los ajustes que se hicieron, tras la aprobación de la Ley 21.298 y a fin de garantizar la representación de los pueblos originarios, se reservaron diecisiete (17) escaños que fueron restados de los siguientes distritos electorales: en la Región Metropolitana, de los distritos 8, 9, 10, 12, 13 y 14; en Bío-Bío, de los distritos 20 y 21 ; en La Araucanía, de los distritos 22 y 23; en Los Lagos, 25 y 26; en Antofagasta, del distrito 3; en Atacama, del distrito 4; en Coquimbo, del distrito 5; en Valparaíso, del distrito 7; y en Los Ríos, del distrito 24. Esta modificación se hizo con base en la mayor proporción de población indígena de más de 18 años en cada distrito, según el censo oficial del INE 2017, sin rebajar más de un escaño por distrito ${ }^{15}$.

La circunscripción para esta representación de los pueblos originarios fue nacional a través de una lista por cada pueblo, diez (10) en total. El número de cargos por elegir es el siguiente: Aimara dos (2); Mapuche ocho (8), Chango uno (1); Colla uno (1); Diaguita uno (1); Kawashkar uno (1); Atacameño uno (1); Quechua uno (1); Rapa Nui uno (1) y Yagán uno (1). El Servicio Electoral (SERVEL) estableció un padrón electoral especial con los electores indígenas y su pueblo indígena de adscripción, con el objeto de permitir este derecho a sufragio; no obstante, dichos electores podían votar o por un candidato de su pueblo

\footnotetext{
${ }^{15}$ Ver https://www.senado.cl/noticias/proceso-constituyente/conozca-de-que-distritos-provendran-los-17-escanosreservados-para
} 


\section{DERECHO ELECTORAL}

indígena, a través de una boleta específica, o por el candidato de su circunscripción electoral. Les estaba permitido un solo voto.

Otro elemento resaltante de la elección del cuerpo constituyente se refirió a políticas cuyo objetivo era garantizar una representación equitativa por género, lo cual significó un avance con respecto a la regulación ya existente, aplicada por primera vez en las elecciones de senadores y diputados al Congreso desde $2017^{16}$. Tras discusiones en el Congreso Nacional, se decidió aprobar, en la Ley 21.216, una representación numéricamente igual por género para la Convención Constitucional, que implicó no solo la obligación de postular de forma paritaria (50\% por género) y alterna (intercalados), aplicable a todos los cargos incluyendo los de representación de pueblos originarios o indígenas, sino, además, la estipulación de un mecanismo de ajuste corrector de las posibles distorsiones de dicho esquema, lo que permitió que el órgano constituyente reflejase una representación equilibrada de setenta y ocho (78) hombres (50\%) y setenta y siete (77) mujeres (50 $\%)^{17}$.

La Ley 21.296 flexibilizó algunos de los dispositivos definidos en la Ley $21.216^{18}$, referidos a la participación de independientes, entre ellos, redujo el porcentaje de firmas para el patrocinio de adhesiones. Las normas definidas para esta elección de la Convención Constitucional permitieron y promovieron la participación de los independientes a través de tres modalidades distintas: además del mecanismo tradicional de estos que tienen un cupo dentro de la lista o bloque partidista, estaban la listas 0 pactos de candidatos independientes y los independientes fuera de lista.

Es de ineludible mención el peso alcanzado por los independientes en este cuerpo: de los 138 convencionales constitucionales electos,

\footnotetext{
16 En la Ley 20.840 se estableció una cuota que implica que ningún sexo podrá superar el $60 \%$ de la totalidad de las candidaturas, independientemente de la forma de nominación de estas, además de incentivos de financiamiento en la campaña electoral.

17 Para más información sobre las políticas de género aplicadas en la elección de la Convención Constitucional, ver de Segovia, Macarena (2021) Convención Constitucional: mecanismos de paridad redujeron a 2,9 \% la brecha de representatividad de las mujeres en https://www.ciperchile.cl/2021/05/17/convencion-constitucional-mecanismos-de-paridad-redujeron-a-29la-brecha-de-representatividad-de-las-mujeres/.

18 Sobre un análisis de la modificación de la Ley 21.216 en cuanto a las candidaturas independientes, ver: http://www.pucv.cl/uuaa/site/docs/20210121/20210121023705/dic_2_ficha_ley_21_296.pdf https://www.cariola.cl/actualidad-constitucional/boletin-constitucional-no2-actualizaciones-en-laconformacion-de-la-convencion-constitucional/
} 


\section{DERECHO ELECTORAL}

excluyendo a los diecisiete (17) representantes que corresponden a los pueblos originarios-, ochenta y ocho (88) son independientes: uno (1) (1 \%) independiente fuera de lista, cuarenta y siete (47) $(34 \%)$ de listas o pactos de candidatos independientes, cuarenta (40) (29\%) dentro de listas o pactos partidistas y solo cincuenta (50) (36 \%) militantes de partidos políticos ${ }^{19}$. La presencia notable de independientes también se observó en las elecciones de gobernadores regionales y de alcaldes ${ }^{20}$.

A la par de estos cambios, se incorporó, en la Ley 21.298, una cuota mínima obligatoria de personas con discapacidad del $5 \%$ del total de candidaturas a ser presentadas por los partidos 0 pactos de partidos ${ }^{21}$, de lo cual resultó electa Adriana Cancino ${ }^{22}$ como convencional constituyente, y se determinó, en la Ley 21.315, la difusión imperativa de franjas electorales televisivas para candidatos a convencionales.

El financiamiento en la campaña electoral es también área de interés en los procesos electorales chilenos. En la elección de convencionales constitucionales hubo preocupación por la cantidad de recursos recaudados, y asimetrías, pese a la fuerte regulación que existe en esa materia en Chile, fundamentalmente la Ley 19.884, norma que también fue aplicada para estos comicios. En la "Lupa Electoral. Informe 2021" se destaca: "La reciente elección de convencionales constitucionales concentró un importante nivel de atención por parte de la ciudadanía y los medios de comunicación, lo cual se relaciona con el alto número de aportes financieros en ellas" (Álvarez, Sajuria y Ortiz, 2021, p. 14).

A dicho tenor es válido reseñar que en el financiamiento de la campaña para la elección de los convencionales se incluyó el dispositivo ya existente de forma temporal para candidatas a senadoras y diputadas al Congreso, referido al reembolso adicional de sus gastos electorales de

\footnotetext{
19 Extraído de Agenda Pública (2021).

${ }^{20}$ En el caso de independientes vencedores para cargos de gobernadores regionales, ver en este artículo, la sección III "Segunda vuelta de las elecciones de gobernadores regionales". En cuanto a las postulaciones para las elecciones de alcaldes 2021, ver https://puentesuc.cl/novedades/elecciones-municipales-2021-unanlisis-de-las-candidaturas; y sobre los escaños obtenidos por partidos e independientes para alcaldes y concejales 2021,

ver

Decide Chile 2021 https://2021.decidechile.cl/\#/ev/2021/ct/2021.A/;(Concejales) https://2021.decidechile.cl/\#/ev/2021/ct/2021.0/.

${ }^{21}$ Para más detalles, ver https://www.bcn.cl/leyfacil/recurso/cupos-para-personas-con-discapacidad-en-laslistas-para-el-proceso-constituyente.

22 Ver https://www.elmostrador.cl/agenda-pais/2021/05/19/chile-pais-inclusivo-solo-una-candidata-ensituacion-de-discapacidad-fue-electa-como-constituyente/.
} 


\section{DERECHO ELECTORAL}

cargo fiscal de 0,01 unidades de fomento por voto obtenido, para el caso de candidatas mujeres; tal prerrogativa se extendió a los candidatos a escaños reservados para pueblos indígenas (Álvarez, Sajuría y Ortiz, 2021).

Dentro de los hallazgos más significativos con respecto al financiamiento electoral en estos $\operatorname{comicios}^{23}$ se tienen las abiertas inequidades evidenciadas en la concentración de los recursos entre los distintos partidos políticos y de estos con respecto al resto de las candidaturas, además de la distribución por género, favorable notablemente al sexo masculino, y el uso de créditos por parte de los más vulnerables para cubrir los gastos de campaña. Esto hace pertinente una revisión de temas como: 1) la reducción de los topes de gastos electorales y de los límites de aportes por personas; 2) el incremento del aporte fiscal y 3 ) las definiciones de políticas afirmativas financieras por género y otros grupos, como los pueblos originarios e independientes, para todos los tipos de elecciones.

En la misma línea, una de las principales preocupaciones relacionadas con los procesos electorales locales en Chile es el uso de los recursos públicos por parte de los alcaldes que van a la reelección ${ }^{24}$ (Contraloría General de la República, 2016; Engel, 2018 y Castellanos, 2019). La Contraloría General de la Republica señaló el incremento sustancial del gasto municipal en los años electorales en el "Estudio sobre Gastos en Publicidad y Difusión en el Sector Municipal 2006" y su preocupación ante el incumplimiento de la recomendación del Consejo Asesor Presidencial contra los conflictos de interés, el tráfico de influencias y la corrupción, en su informe de 2015, de limitar a un $5 \%$ el aumento del gasto en publicidad y difusión en un año electoral respecto de los tres años precedentes. Según la Contraloría General de la República: "Solo un $29 \%$ de los municipios habría cumplido con la recomendación de dicho Consejo, mientras que un $68 \%$ de los municipios lo habría sobrepasado" (2016, p. 5).

\footnotetext{
23 Ver, también, Vásquez, Agustín (2021) Desigualdad en campaña: las vías de financiamiento que favorecen a los partidos tradicionales en https://plataformacontexto.cl/contexto_factual/desigualdad-encampana-las-vias-de-financiamiento-que-favorecen-a-los-partidos-politicos-tradicionales/ y Sepúlveda Nicolás, Cifuentes Lucas y Ríos Cristóbal (2021) en https://www.ciperchile.cl/2021/05/17/eleccionconstituyente-las-campanas-millonarias-que-fueron-derrotadas-por-candidaturas-casi-sin-fondos/.

${ }^{24}$ Los alcaldes que van a la reelección deben separarse temporalmente de sus cargos treinta (30) días antes de la realización de la elección, con las prerrogativas que determina la Ley 18.695, ver https://www.bcn.cl/leychile/navegar?idNorma=251693.
} 


\section{DERECHO ELECTORAL}

Castellanos recalca con respecto al financiamiento electoral en las campañas de elecciones locales en Chile:

Evaluar el financiamiento electoral en las elecciones de Alcaldes resulta clave para comprender más exhaustivamente las asimetrías entre los incumbentes y los desafiantes. Existe la preocupación de que dadas las últimas reformas en las cuales se redujo el financiamiento electoral privado, las autoridades que se someten a la reelección, especialmente los Alcaldes, por poseer grandes recursos gracias al ejercicio del cargo a la par de los limitados mecanismos de control en las municipalidades, corran con ventaja en la contienda comicial. (2019, p. 24).

Previo a la celebración de estas elecciones locales de 2021, la Contraloría General de la República emitió el dictamen E50319, de fecha 10 de noviembre de 2020, en el que se destacan, entre otros, la debida probidad administrativa en el ejercicio de sus funciones, así como la prohibición de involucrarse en la campaña electoral durante la jornada laboral y del uso de bienes, vehículos y recursos físicos y financieros en actividades políticas. Habrá que esperar estudios a posteriori que determinen cómo fue el comportamiento de los alcaldes a este tenor en los comicios de 2021.

Es de acotar que el número de mandatos y posibilidades de reelección en Chile sufrió una modificación sustancial en 2020, con la Ley 21.238, al limitar a los senadores a un máximo de un período adicional; y a los diputados al Congreso, consejeros regionales, alcaldes y concejales, a un máximo de dos períodos adicionales. Para el caso de los gobernadores regionales se mantuvo un solo período adicional sucesivo.

Chile incorporó cuantiosos protocolos biosanitarios a los fines de asegurar que las jornadas de votación de las diferentes elecciones celebradas durante el año 2021, al igual que en 2020, no significasen un riesgo desde el punto de vista de propagación de la pandemia, especialmente porque su realización se inserta en tiempos de incremento de los casos de contagio y de diferentes niveles de cuarentena (Ministerio de Salud, 2020). Entre las medidas ${ }^{25}$ se

\footnotetext{
25 Para la revisión comparada de medidas en el manejo del COVID-19 en elecciones, recomendamos IDEA Internacional. Panorama global del impacto del COVID-19 en las elecciones en https://www.idea.int/es/news-media/multimedia-reports/panorama-global-del-impacto-del-covid-19-en-laselecciones.
} 


\section{DERECHO ELECTORAL}

encuentran ${ }^{26}$, además del novedoso y poco frecuente esquema de dos días continuos de votación para las Elecciones de la Convención Constitucional ${ }^{27}$, gobernadores regionales y cargos municipales los días 15 y 16 de mayo 28 ; uso de las mascarillas; distanciamiento de por lo menos un metro; extensión del horario de votación; kit de elementos de protección personal para los que desarrollen labores electorales; solicitud, no imperativa, al elector de portar su lápiz para el ejercicio de voto; recomendación de horario preferencial para personas de la tercera edad o con condiciones especiales; ampliación del número de locales de votación; prohibición de participar a personas contagiadas con COVID19 o con un contacto positivo estrecho; uso de alcohol en gel; ausencia de puerta o cortina en la cámara secreta y elementos de desinfección, uso de guantes y escudo facial para el momento del conteo de votos, entre otros.

La participación electoral de las elecciones del 15 y 16 de mayo de 2021 fue de 6468750 electores. Además de la reducción de la concurrencia con respecto al Plebiscito Nacional de 2020, de un 50,95 \% a un 43,41 $\% 29$, se constató una composición diversa en cuanto a la pluralidad de partidos independientes, a lo que debe sumarse, para el caso de la Convención Constitucional, la representación equitativa por género ${ }^{30}$ y la cuota de pueblos originarios ${ }^{31}$ (ver figura 1 ).

\footnotetext{
${ }^{26}$ Sobre protocolos COVID 2019 en elecciones en Chile 2021, ver Elecciones del 15 y 16 de mayo 2021 en https://www.servel.cl/wp-

content/uploads/2021/04/SERVICIO_ELECTORAL_PROTOCOLO_SANITARIO_ELECCIONES_2021_-

_ACUERDO_24_MARZO_VB_MINSAL.pdf y Elecciones del 21 de noviembre 2021 en https://servel.cl/wpcontent/uploads/2021/10/SERVICIO_ELECTORAL_PROTOCOLO_SANITARIO_ELECCIONES_GENERALES_202 1_ACUERDO_6_de_OCT_vf.pdf

${ }^{27}$ En la Ley 21.317 (https://www.bcn.cl/leychile/navegar?idNorma=1157063), se estableció que se llevaría a cabo durante dos días, 10 y 11 de abril, la celebración de las elecciones de gobernadores regionales, alcaldes, concejales y convencionales constitucionales. En la Ley 21.324 , se prorrogaron las fechas al 15 y 16 de mayo.

${ }^{28}$ Vale destacar que el conteo solo se realizaría el segundo día de las votaciones. El primer día al finalizar la jornada se cerrarían las cajas y quedarían en custodia de las Fuerzas Armadas.

${ }_{29}$ Si bien constituye una participación menor con respecto al Plebiscito Nacional de 2020, este valor es similar a las elecciones locales de 2012 y superior al de las de 2016, y se inscribe en el esquema de reducción de la participación, tras la adopción del voto voluntario en 2012. Para detalle de la participación electoral en Chile desde 1988 a 2021, ver PNUD (2021) Participación electoral y voto obligatorio en file://C:/Users/Usuario/Downloads/CL\%20Reimplementaci\%C3\%B3n\%20voto\%20obligatorio\%20Analisis\% 20PNUD\%2031\%20mayo\%202021.pdf

${ }^{30}$ En las elecciones de instancias regionales y municipales no están contempladas políticas de género, como existen para los cargos de senadores y diputados al Congreso.

31 Para más información sobre los resultados electorales de las elecciones del 15 y 16 de mayo, por tipo de elección, ver https://www.servel.cl/resultados-definitivos-elecciones-de-convencionales-constituyentesgobernadores-regionales-alcaldes-y-concejales/
} 


\section{DERECHO ELECTORAL}
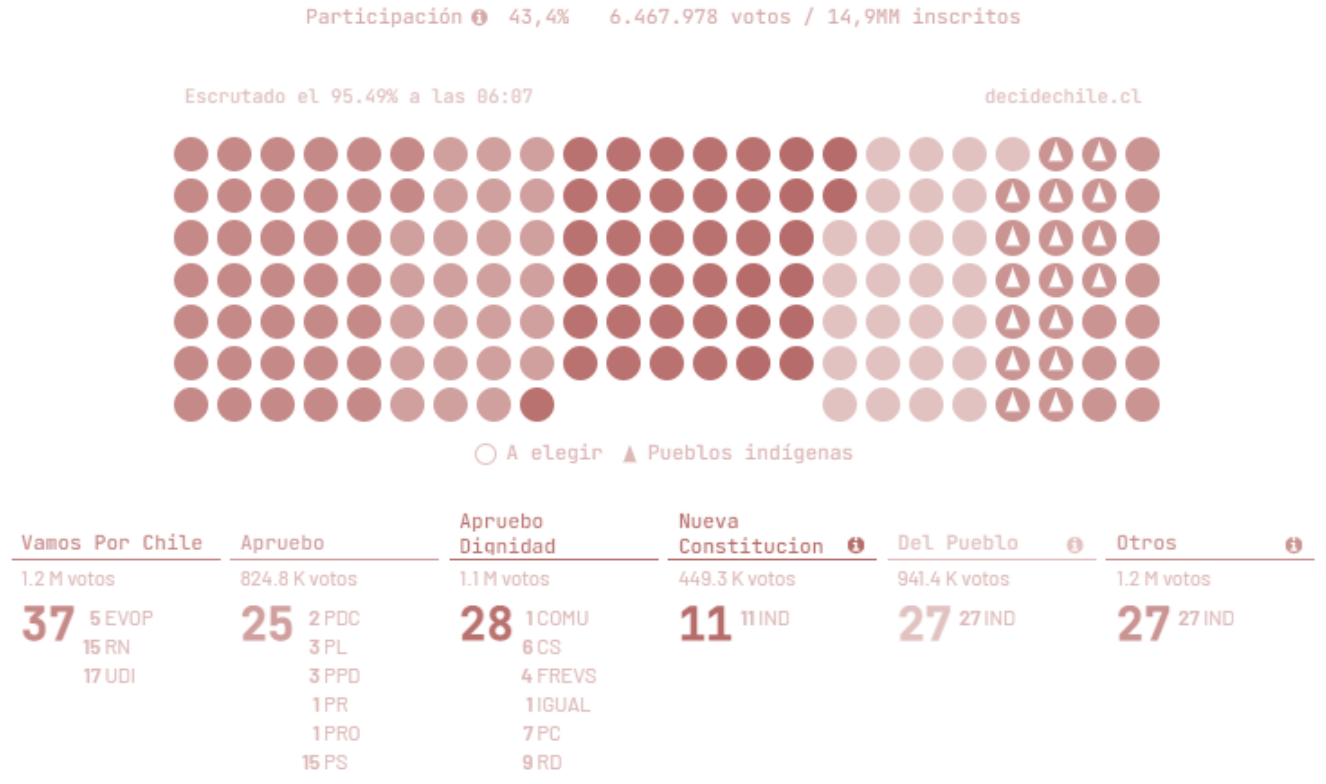

Figura 1. Elecciones 2021. Constituyentes. Tomado de Decide Chile (2021).

No es de menor significancia lo expuesto por Andrés Tagle, presidente del Consejo Directivo del Servicio Electoral (SERVEL), como posibles causas de la baja participación en estos comicios: 1) Fallas en la formación y actualización del registro electoral, específicamente con respecto a los domicilios desactualizados, esto entendido como personas inscritas en comunas donde no residen actualmente. Tagle afirmó: "Estimamos que hay una desactualización del domicilio electoral que va de $25 \%$ al $30 \% "$ (Wilson, 2021, párr. 3); 2) el caso de los chilenos fuera del territorio nacional que no han actualizado sus datos y 3) los fallecidos cuyos registros aún no han sido depurados (Wilson, 2021).

Resultan preocupantes las debilidades en la actualización de los datos en el registro electoral, dentro y fuera del territorio nacional, dado que desestimula la concurrencia a elecciones, y que, al igual que la figura de voto voluntario (Ríos, 2021), pudiera convertirse en uno de los posibles factores explicativos de la abstención electoral. La actualización y la 


\section{DERECHO ELECTORAL}

depuración efectiva del registro electoral son parte fundamental de cualquier elección $y$, hoy por hoy, es uno de los principales retos electorales de Chile.

No es posible concluir un análisis sobre las elecciones del 15 y 16 de mayo de 2021 sin mencionar que, salvo el caso de sesenta y cinco (65) mesas receptoras de sufragio de cinco (5) locales, correspondientes a la Comuna de San Ramón en la Región Metropolitana de Santiago ${ }^{32}$, en donde hubo necesidad de repetir las elecciones por presuntas irregularidades ocurridas durante la jornada de votación; los demás resultados electorales fueron reconocidos y certificados, lo cual pone en evidencia la cultura democrática del pueblo chileno, así como la fortaleza de la institucionalidad comicial y de las fuerzas armadas en la organización y la custodia de los procesos electorales, respectivamente.

\section{Segunda vuelta de las Elecciones de Gobernadores Regionales}

El 13 de junio de 2021, fue convocada finalmente la segunda vuelta de los gobernadores regionales en aquellas entidades donde ninguno de los candidatos a dicho cargo hubiese alcanzado el $40 \%$ de los votos válidos emitidos en las elecciones del 15 y 16 de mayo. De las dieciséis (16) regiones, trece (13) fueron a segunda vuelta ${ }^{33}$. Los tres vencedores en las elecciones de mayo fueron:

- Valparaíso: Rodrigo Mundaca (Frente Amplio).

- Aysén: Andrea Macías (Partido Socialista) (Unidad Constitucional).

- Magallanes: Jorge Files (Independiente Partido Radical) (Unidad Constituyente).

La participación electoral fue de 2558962 votantes, un 19,62 \% del padrón, lo que implica una reducción sustancial con respecto a

\footnotetext{
32 Para más información sobre la repetición de elecciones en la Comuna de San Ramón de la Región Metropolitana de Santiago, ver https://www.servel.cl/servel-explico-como-sera-la-repeticion-de-eleccionesmunicipales-en-san-ramon/

33 Para más información sobre la segunda vuelta de las Elecciones de gobernadores regionales 2021, ver https://elecciones2021.servel.cl/segunda_votacion_gores/ y https://elecciones2021.servel.cl/wpcontent/uploads/2021/06/1948621.pdf
} 


\section{DERECHO EIECTORAL}

las elecciones del 15 y 16 de mayo (43,41 \%). He aquí los resultados en el resto de las regiones de la segunda vuelta ${ }^{34}$ :

- Arica y Parinacota: Jorge Díaz Ibarra (Partido Demócrata Cristiano) (Unidad Constituyente).

- Tarapacá: José Miguel Carvajal (Partido Comunes) (Frente Amplio).

- Antofagasta: Ricardo Díaz (Independiente Partido Progresista) (Unidad Constituyente).

- Atacama: Miguel Vargas (Independiente).

- Coquimbo: Krist Naranjo (Independiente).

- Metropolitana: Claudio Orrego (Partido Demócrata Cristiano) (Unidad Constituyente).

- O'Higgins: Pablo Silva (Partido Socialista) (Unidad Constituyente).

- Maule: Cristina Bravo (Partido Demócrata Cristiano) (Unidad Constituyente).

- Ñuble: Óscar Crisóstomo (Partido Socialista) (Unidad Constituyente).

- Bío: Rodrigo Díaz (Independiente).

- La Araucanía: Luciano Rivas (Independiente Evolución Política) (Chile Vamos).

- Los Ríos: Luis Cuvertino Gómez (Partido Socialista) (Unidad Constituyente).

- Los Lagos: Patricio Vallespín (Partido Demócrata Cristiano) (Unidad Constituyente).

\section{Primarias PResidenciales: Oficial y Convencional}

\section{a) Primarias presidenciales}

Para el año 2021, estaba contemplada, de igual forma, la realización de primarias presidenciales, cuya fecha también fue postergada. Al final, solo dos pactos o bloques participaron el 18 de julio en las primarias presidenciales oficiales organizadas por el Servicio Electoral (SERVEL); por un lado, los partidos oficialistas (centro derecha), agrupados en el Pacto "Chile Vamos": Unión Demócrata Independiente (UDI),

\footnotetext{
34 Resultados electorales de la segunda vuelta de gobernadores regionales 2021 (https://www.servel.cl/resultados-provisorios-segunda-votacion-de-gobernadores-regionales-2021/)
} 


\section{DERECHO ELECTORAL}

Renovación Nacional (RN) y Evolución Política (EVOPOLI); y por el otro, el Pacto Apruebo Dignidad (izquierda) conformado por el Partido Comunista (PC) y el Partido Frente Amplio (FA).

Podían votar en estas primarias presidenciales los afiliados a los partidos o pactos que se sometieran a dicha elección, así como los independientes sin afiliación política habilitados para ejercer el derecho al sufragio tanto en Chile como en el extranjero. Los afiliados a partidos políticos solo podían votar en la primaria del pacto al que correspondiese su partido, mientras que los independientes recibían una cédula única con los candidatos de ambos pactos y debían votar por solo uno de ellos (Chile, 2017).

En el caso de la elección primaria del Pacto Chile Vamos, un representante por cada uno de los partidos de la alianza postuló su candidatura: Joaquín Lavín (UDI), Ignacio Briones (EVOPOLI) y Mario Desborde (RN), además del independiente Sebastián Sichel. En cuanto al Pacto Apruebo Dignidad, se postularon Gabriel Boric como candidato del FA y Daniel Jadue por el PC. A tono con el mandato constitucional y legal vigente, se contempló para estas primarias la transmisión obligatoria de la franja electoral del 30 de junio al 15 de julio de 2021, ambos días inclusive (Consejo Nacional de Televisión de Chile, 2021).

Estas primarias lograron una participación histórica en Chile, en las cuales ascendió a 3139765 el número de electores, dentro y fuera $^{35}$ del territorio nacional. El Pacto Chile Vamos obtuvo 1340 020 votos válidos, y 1751 759, el Pacto Apruebo Dignidad. Los resultados fueron ${ }^{36}$ : por el Pacto Apruebo Dignidad, Gabriel Boric 1058027 (60,43\%) y Daniel Jadue 692862 (39,57\%). Por el Pacto Chile Vamos, Sebastián Sichel 659416 (49,08 \%), Joaquín Lavín 420 691 (31,30\%), Ignacio Briones 131957 (9,82\%) y Mario Desbordes con $131674(9,80 \%)$.

Es de obligatoria mención los pronósticos electorales reflejados en la mayoría de las encuestas difundidas durante las primarias presidenciales de 2021, entre ellas, las de CADEM Research \& Estrategia, la encuestadora más difundida en el país austral. De acuerdo

\footnotetext{
35 Para las primarias presidenciales, así como las elecciones presidenciales y plebiscitos nacionales, los chilenos en el exterior pueden ejercer su derecho al voto. Más información al respecto en https://www.servel.cl/voto-exterior/.

${ }^{36}$ Resultados electorales de las primarias presidenciales 2021 en https://www.servelelecciones.cl/.
} 
a los sondeos de CADEM ${ }^{37}$, hasta la fecha en que se les permitió publicar sondeos de opinión, los candidatos vencedores serían Joaquín Lavín por Chile Vamos y Daniel Jadue por Apruebo Dignidad. En ninguno de los casos se cumplieron las predicciones, lo cual no es novedoso; basta solo recordar lo ocurrido con la abanderada presidencial por el Frente Amplio, Beatriz Sánchez, en las Elecciones Generales Nacionales del año 201738. Las fallas de predicción pueden responder a múltiples factores ${ }^{39}$, entre otros, deficiencias en la metodología o cambios de opinión de los electores en el lapso de prohibición de publicación de las encuestas, de allí que sea recomendable revisar su regulación ${ }^{40}$ en aras de una potencial reforma electoral en temas como patrocinio, publicación de información sobre el universo de la muestra y la muestra en sí, etc.

\section{b) Primarias convencionales presidenciales}

Partidos políticos de centro izquierda que integran el Pacto Unidad Constituyente y que no lograron participar en las primarias oficiales presidenciales convocaron a una consulta denominada "Primaria Convencional", para el 21 de agosto de 2021. Este proceso no fue organizado por el Servicio Electoral (SERVEL).

\section{Para estos comicios podían participar los militantes de los partidos que conforman el bloque Demócrata Cristiano (DC), Por la Democracia}

\footnotetext{
37 CADEM en su cuenta de Twitter publicó el día 18 de julio, luego de conocidos los resultados de las primarias presidenciales, dos tweets en donde señalaba que en la última medición las proyecciones cambiaban y se ajustaban a la votación de las primarias y que no pudo ser publicada por la prohibición de ley (Ley 18.700). Ver tweet sobre resultados de las primarias de Pacto Apruebo Dignidad: https://twitter.com/Cadem_cl/status/1416913485657743364?s=20 y tweet sobre resultados de las primarias de Pacto Chile Vamos: https://twitter.com/Cadem_cl/status/1416912775545344000?s=20.

38 Se recomienda la lectura de la opinión de Navia, Patricio y Espinoza, Gonzalo "En qué se equivocó la encuesta de CADEM" publicada el 12/12/2017 (https://www.ciperchile.cl/2017/12/12/en-que-se-equivocola-encuesta-cadem/).

39 Un análisis sobre razones posibles de las fallas de las encuestas electorales en Chile se encuentra en Sherman, Andrés "Cuatro posibles razones de la imprecisión de las encuestas electorales", publicado el $26 / 07 / 2021$ encuestas-electorales/).

40 Para más información en torno a regulación comparada sobre encuestas electorales, recomendamos el artículo de Marshall, Pablo; Charney, John y Rosas, Nelson (2021) Regulación legal de las encuestas electorales: análisis teórico

(http://www.scielo.org.mx/scielo.php?script=sci_arttext\&pid=S1405-91932020000100215); Gabriela (2020), "Regulación encuestas electorales. Experiencia comparada" (https://www.camara.cl/verDoc.aspx?prmID=216695\&prmTipo=DOCUMENTO_COMISION); Orr, Graeme y Levy, Ron (2016) Regulating opinion polling: A deliberative democratic perspective. Ver https://www.unswlawjournal.unsw.edu.au/wp-content/uploads/2017/09/39-1-5.pdf.
} 


\section{DERECHO ELECTORAL}

(PPD), Radical (PR), Socialista (PS), Progresista (PRO), Liberal (PL) y Nuevo Trato y Ciudadanos; así como los electores sin afiliación partidista. Se utilizaron como centros de votación los mismos locales habilitados para las primarias de gobernadores regionales celebradas en el año 2020.

Los candidatos presentados fueron Yasna Provoste por el Partido Demócrata Cristiano (PDC), Paula Narváez por los partidos Socialista (PS), Por la Democracia (PPD) y Nuevo Trato, y Carlos Maldonado por el Partido Radical (PR). Con una votación de un poco más de 150000 votos válidos (150 881), Yasna Provoste alcanzó 91789 votos, Paula Narváez 40161 y Carlos Maldonado 18931.

\section{Elecciones Presidencial, Senadores, Diputados al Congreso $Y$ Diputados a los Consejos Regionales}

Las elecciones para escoger al presidente de la República, senadores y diputados al Congreso y consejeros regionales ${ }^{41}$ se realizaron el 21 de noviembre, fecha que no sufrió postergación. El padrón electoral ascendió a $15030963^{42}, 14959945$ electores en el territorio nacional y 71018 fuera de Chile ${ }^{43}$. Se escogieron, además del jefe del ejecutivo nacional, veintisiete (27) senadores, en una renovación parcial de la cámara ${ }^{44}$, la totalidad de los diputados al Congreso ${ }^{45}$, ciento cincuenta y cinco (155), así como trecientos dos (302) consejeros regionales ${ }^{46}$. Los chilenos inscritos en el registro electoral fuera del territorio nacional solo pudieron participar en la elección del presidente de la República. La tabla 2 presenta la distribución del número de cargos por elegir en las elecciones del 21 de noviembre de 2021.

\footnotetext{
${ }^{41}$ Para más información sobre los cargos de elección popular en Chile: (sistema electoral, circunscripción, periodo de mandato, requisitos), ver: https://formacionciudadana.servel.cl/usuarios/113/lista-contenido 42 Información sobre el padrón electoral definitivo. Elecciones Generales 2021.

https://www.servel.cl/padron-electoral-definitivo-y-nomina-de-inhabilitados-elecciones-generales-2021/

${ }^{43}$ Sobre los centros de votación en el exterior. Elecciones Generales 2021. https://www.servel.cl/wpcontent/uploads/2020/07/Copia_de_circunscripciones_web_servel_2.pdf

${ }^{44}$ La renovación parcial del Senado comenzó en las Elecciones Generales de 2017, de conformidad con la Ley 20.840. En las elecciones de 2021 se elegirán senadores en las siguientes regiones: Antofagasta, Coquimbo, O'Higgins, Nuble, Biobío, Los Ríos, Los Lagos, Magallanes y Metropolitana.

${ }^{45}$ Información sobre los candidatos postulados para presidente de la República, senadores, diputados al Congreso 2021 en https://presidenciales2021.servel.cl/wp-content/uploads/2021/09/BoletinUnico-PRESSEN-DIP.pdf.
}

46 Información sobre los candidatos postulados para consejeros regionales https://presidenciales2021.servel.cl/wp-content/uploads/2021/10/Boletin-Unico-Consejerosas-

Regionales.pdf. 


\section{DERECHO EIECTORAL}

\section{Tabla 2}

Cargos por elegir. Elecciones Generales noviembre, 2021

\begin{tabular}{|c|c|c|c|}
\hline Región & $\begin{array}{c}\mathbf{N} .^{\circ} \text { de } \\
\text { senadores por } \\
\text { elegir }^{\mathrm{a}}\end{array}$ & $\begin{array}{c}N^{\circ}{ }^{\circ} \text { de } \\
\text { diputados por } \\
\text { elegir }\end{array}$ & $\begin{array}{c}\mathbf{N} .^{\circ} \text { de } \\
\text { consejeros } \\
\text { regionales por } \\
\text { elegir }{ }^{c}\end{array}$ \\
\hline Arica y Parinacota & No elige & 3 & 14 \\
\hline Tarapacá & No elige & 3 & 14 \\
\hline Antofagasta & 2 & 5 & 16 \\
\hline Atacama & No elige & 5 & 14 \\
\hline Coquimbo & 3 & 7 & 16 \\
\hline Valparaíso & No elige & 16 & 28 \\
\hline $\begin{array}{l}\text { Metropolitana de Santiago } \\
\text { Libertador Bernardo O'Higgins }\end{array}$ & $\begin{array}{l}5 \\
3\end{array}$ & $\begin{array}{c}47 \\
9\end{array}$ & $\begin{array}{l}34 \\
20\end{array}$ \\
\hline $\begin{array}{l}\text { Del Maule } \\
\text { De Ñuble }\end{array}$ & $\begin{array}{c}\text { No elige } \\
2\end{array}$ & $\begin{array}{c}11 \\
5\end{array}$ & $\begin{array}{l}20 \\
16\end{array}$ \\
\hline $\begin{array}{l}\text { Del Biobio } \\
\text { De la Araucanía } \\
\text { De los Ríos }\end{array}$ & $\begin{array}{c}3 \\
\text { No elige } \\
3\end{array}$ & $\begin{array}{c}13 \\
11 \\
5\end{array}$ & $\begin{array}{l}28 \\
20 \\
14\end{array}$ \\
\hline De los Lagos & 3 & 9 & 20 \\
\hline De Aysén & No elige & 3 & 14 \\
\hline $\begin{array}{l}\text { De Magallanes y la Antártica } \\
\text { Chilena }\end{array}$ & 3 & 3 & 14 \\
\hline
\end{tabular}

$\begin{array}{llll}\text { Total de cargos por elegir } & \mathbf{2 7} & \mathbf{1 5 5} & \mathbf{3 0 2}\end{array}$

Nota: Elaborado con base en datos recopilados del Servicio Electoral de Chile

${ }^{a}$ El número de senadores por elegir en cada región es proporcional a la población, en un rango que oscila entre 2 y 5 (Ley 20.840). ' El número de diputados a elegir por cada región es proporcional a la población, en un rango que oscila entre 3 y 8 (Ley 20.840). ' El número de consejeros regionales a elegir por cada región es proporcional a la población, en un rango que oscila entre 14 y 34 (Ley 20.678 https://www.bcn.cl/leychile/navegar?idNorma=1051270).

Los sistemas electorales aplicados son de mayoría absoluta ${ }^{47}$ para el presidente de la República y de representación proporcional para senadores, diputados al Congreso y consejeros regionales, a través del método de divisores de D'Hondt. Las circunscripciones en el caso de senadores son según la división político-territorial (regiones), con un tamaño proporcional a la población, mientras que, en el caso de

\footnotetext{
47 "Mientras que para Presidente de la República se aplica una mayoría absoluta, es decir, la mitad más uno de los votos válidos emitidos, para los Gobernadores Regionales se requiere un porcentaje mínimo (40\%) y para los Alcaldes se exige solo una mayoría simple o relativa.(En todos los casos se excluyen para el escrutinio los votos en blanco y nulos). (Castellanos, 2019, p. 23). La reelección para el caso del presidente de la República no puede ser inmediata y es sin límites (Constitución Política de la República de Chile. Recuperado de https://www.bcn.cl/leychile/navegar?idNorma=242302).
} 


\section{DERECHO ELECTORAL}

diputados al Congreso y consejeros regionales, si bien también son fijadas de forma proporcional a la población, estas pueden ser, para el caso de diputados, según la división político-territorial o fijadas artificialmente, $y$, en el caso de consejeros regionales, fijadas artificialmente. En todos los casos son de tamaño variable no uninominal. Se mantienen las políticas de acción afirmativa a favor del género femenino (cuota, incentivos en la postulación) instrumentadas a partir de 2017, para candidatas a senadoras y diputadas al Congreso Nacional 48 .

En las elecciones el elector contó con cuatro votos para ejercerlos en cuatro cédulas o boletas electorales diferentes ${ }^{49}$. Una para elegir a un candidato a la presidencia de la República, una para elegir a un candidato al Senado, una para elegir a un candidato a la Cámara de Diputados al Congreso y una para elegir a un candidato al Consejo Regional. Todos los votos son uninominales.

A la presidencia de la República se postularon siete (7) candidatos. Si bien no es el mayor número de opciones en una elección de esta naturaleza en Chile desde 1989 (Bravo, 2021), año del retorno de elecciones democráticas posterior a la dictadura de Pinochet, se infirió, al analizar la pugnacidad política, polarización, sensibilidad sociopolítica tras las protestas de 2019 y temas como las Administradoras de Fondos de Pensiones (AFP) y el COVID-19, así como la dinámica de la campaña electoral y lo que arrojaron los estudios de opinión ${ }^{50}$, que sería un resultado con una alta fragmentación del voto. Los candidatos fueron ${ }^{51}$ :

-Gabriel Boric Font, por el Pacto Apruebo Dignidad

-Antonio Kast Rist, por el Partido Republicano de Chile

-Yasna Provoste Campillay, formalmente por el Partido Demócrata Cristiano (ver Primarias Convencionales 2021)

- José Sebastián Sichel Ramírez, por el Pacto Chile Vamos

-Eduardo Artés Brichetti, por el Partido Unión Patriótica

\footnotetext{
48 Para más información sobre las políticas de género en Chile en 2017 se recomienda: PNUD. (2018)

$49 \mathrm{He}$ aquí los facsímiles de las boletas electorales. Elecciones del 21 de noviembre de 2021. https://www.servel.cl/facsimiles-de-cedulas-elecciones-presidenciales-parlamentarias-y-de-consejerosregionales-2021/

50 Para ampliar ver Sondeos de intención de voto para la elección presidencial de Chile de 2021, en https://es.wikipedia.org/wiki/Anexo:Sondeos_de_intenci\%C3\%B3n_de_voto_para_la_elecci\%C3\%B3n_pres idencial_de_Chile_de_2021.

51 Para información de los candidatos presidenciales 2021 y sus programas de gobierno, ver https://presidenciales2021.servel.cl/candidaturas-y-pactos/.
} 


\section{DERECHOELECTORAL}

- Marco Enríquez-Ominami Gumucio, por el Partido Progresistas de Chile

-Franco Parisi Fernández, por el Partido de la Gente

La campaña electoral en Chile es entendida, conforme a la Ley 18.700, como el lapso preparatorio y de ejecución de los actores postulantes y/o postulados en lo concerniente a la planificación y organización de equipos, búsqueda de recursos financieros, generación de gastos electorales y agendas de candidatos en reuniones, visitas, etc. Comienza el día que vence el plazo para la declaración de las candidaturas y se extiende hasta el día de la elección. Al formalizarse las candidaturas y antes de iniciarse el lapso de la propaganda electoral, la oferta electoral puede realizar actividades que no signifiquen propaganda electoral, tales como reuniones con adherentes, visitas al electorado, etc. La propaganda electoral, por su parte, comprende los eventos y/o manifestaciones públicas; así como las publicidades radiales, escritas, en imágenes, en soportes audiovisuales u otros medios análogos en las cuales se promuevan candidaturas y/o partidos con fines electorales. Se plantean en diferentes momentos dependiendo del tipo de propaganda (ver figura 2).

\section{GENERALESO21 $=$}

Línea de tiempo: Períodos de "Campaña Electoral" y "Propaganda Electoral" Elecciones de Presidente de la República, Senadores, Diputados y Consejeros Regionales 2021.

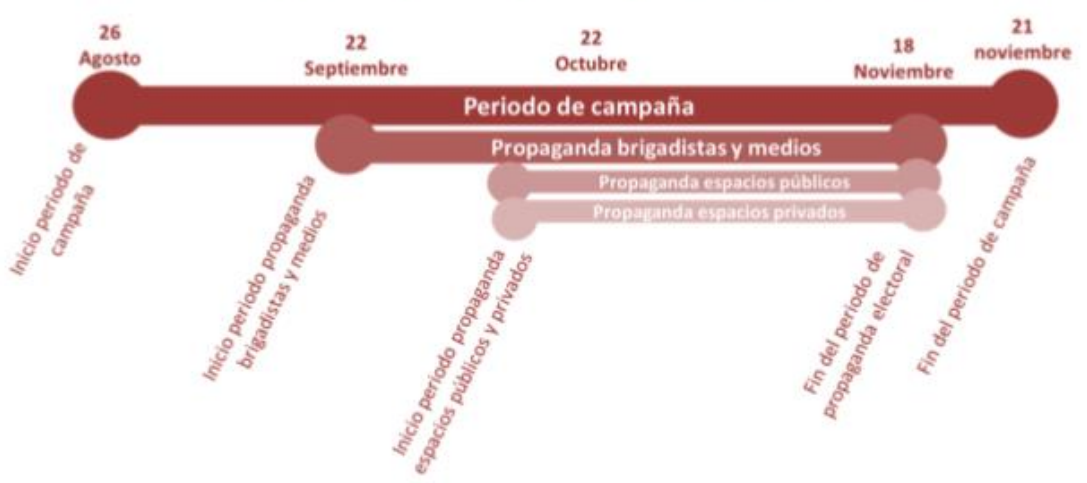

Figura 2. Campaña y propaganda electoral. Elecciones Generales Chile 2021. Recuperado de SERVEL. Manual de publicidad y propaganda electoral. Elecciones Generales 2021. 


\section{DERECHO ELECTORAL}

Los principales lapsos de la campaña y la propaganda electoral son, según el Servicio Electoral (2020):

- Inicio de la campaña electoral: 23-08-2021.

- Inicio de período de propaganda electoral en prensa escrita, radioemisoras y medios digitales y mediante activistas 0 brigadistas en la vía pública: 22-09-2021.

- Inicio de período de propaganda electoral mediante carteles, afiches y letreros en espacios públicos y privados: 22-10-2021.

- Inicio de período de prohibición de divulgar resultados de encuestas sobre preferencias electorales: 06-11-2021.

- Fin de ambos períodos de propaganda. A partir de las 23:59 horas de esta fecha queda prohibida la realización de cualquier tipo de propaganda electoral: 18-11-2021.

Sobre las redes sociales existen al menos tres reglas básicas: 1) Los candidatos y los partidos pueden contratar anuncios en páginas web y redes sociales los cuales deberán sujetarse a las disposiciones de la Ley 19.884. 2) Las expresiones e interacciones realizadas desde las cuentas personales de los candidatos y/o sus adherentes, y por cuya difusión no se pague a la plataforma respectiva, se contemplan como parte de la libertad de información y de opinión, por lo tanto, no se consideran propaganda electoral. Y 3) Los organismos públicos que dispongan de medios de comunicación social, de cualquier tipo, físico y/o digital, no podrán destinar sección o espacio alguno de estos para realizar propaganda electoral, proselitismo, ni favorecer o perjudicar a cualquier candidatura o partido político.

Las recomendaciones del Servicio Electoral (SERVEL) para la campaña electoral en cuanto a protocolos biosanitarios para las Elecciones Generales de noviembre, al igual que para las del 15 y 16 de mayo (Servicio Electoral de Chile, 2020) fueron: no realizar actos masivos, preferir los canales digitales para la divulgación de ideas y planes y cumplir con la distancia física en las reuniones presenciales, entre otros.

Dos de los elementos que más acaparan la atención durante las campañas electorales presidenciales en Chile son las franjas electorales televisivas y los debates presidenciales. Las primeras están reguladas en la Ley 18.700. De acuerdo al Manual sobre Publicidad y Propaganda Electoral. Elecciones de setiembre de 2021: 


\section{DERECHO EIECTORAL}

Los candidatos tendrán derecho desde 30 días y hasta el tercer día anterior a la elección, a un espacio de franja gratuita de televisión. Es decir, desde el viernes 22 de octubre hasta el 17 de noviembre 2021. De acuerdo al artículo 32 de la ley N¹8.700, los canales de televisión abierta deberán destinar gratuitamente un espacio de 40 minutos, de los cuales 20 minutos se destinarán a la elección de Presidente y los restantes 20 minutos se distribuirán entre las campañas para elecciones de Senadores, Diputados y Consejeros Regionales. Para el caso de Presidente de la República, los 20 minutos deberán distribuirse en partes iguales entre los candidatos participantes. Para el caso de los Senadores, Diputados y Consejeros Regionales, los tiempos de [sic] distribuirán de acuerdo a la votación obtenida por cada partido en la última elección de Diputados. Los partidos políticos que no hubieren participado en la última elección de Diputados obtendrán un tiempo igual al obtenido por el partido de menor votación. Las candidaturas independientes, en su conjunto, recibirán un tiempo igual al partido que hubiere obtenido la menor votación, el que se distribuirá en partes iguales entre ellas. La franja electoral, tanto en su contenido como en su distribución, es regulada por el Consejo Nacional de Televisión (CNTV). (2021, p. 21).

En cuanto a los debates, hasta noviembre se habían realizado ocho, en los que se discutieron grandes temas nacionales como seguridad social, vivienda, empleo, educación, protección a la niñez, salud, agua, migración, pueblos indígenas, política exterior, entre otros.

- Debate presidencial del 22 de setiembre de 2021, link https://youtu.be/y1uxdnWeKUU

- Debate Confederación de la Producción y Comercio del 24 de octubre de 2021, link https://youtu.be/y1uxdnWeKUU

- Debate presidencial del 11 de octubre de 2021, link https://youtu.be/y1uxdnWeKUU

- Debate de la Asociación de radiodifusores de Chile de 15 de octubre de 2021, link https://youtu.be/LqO5mcTkI_s

- Debate futuro del agua de Chile, del 21 de octubre de 2021,link https://www.youtube.com/watch?v=FGxg-Q6giqs 


\section{DERECHO ELECTORAL}

- Debate Universidad de Chile del 1 de noviembre de 2021, link https://www.youtube.com/watch?v=FGxg-Q6giqs

- Debate Ciudad 2030, del 4 de noviembre de 2021, link https://www. youtube.com/watch?v=FGxg-Q6giqs

- Debate sobre el medio ambiente del 5 de noviembre de 2021, link https://www.youtube.com/watch?v=FGxg-Q6giqs

La ausencia en territorio nacional del candidato presidencial Franco Parisi ${ }^{2}$, quien se encuentra actualmente residenciado en los Estados Unidos, resulta un hecho curioso y preocupante dentro de la campaña electoral. La legislación electoral en Chile no consagra expresamente la obligación de estar presente en la campaña, ni siquiera asistir al acto de votación, aunque ello pudiera entenderse como contrario al concepto de una verdadera oferta electoral.

A tono con la situación política de Chile, la campaña fue de fuertes confrontaciones $y$, de acuerdo a lo que arrojan los estudios de opinión, existiendo una aparente polarización de las preferencias electorales entre José Antonio Kast, el candidato de derecha y simpatizante de la dictadura de Augusto Pinochet y Gabriel Boric, del Pacto de izquierda Apruebo Dignidad, el cual incluye al Partido Comunista, aunque ninguno logró la mayoría absoluta necesaria para alcanzar la presidencia de la República. Dado lo anterior, el 19 de diciembre se celebraró la segunda vuelta con los candidatos José Antonio Kast de extema derecha y Gabriel Boric de izquierda, donde resultó electo Boric.

\section{A mANERA DE CONCLUSiONeS}

Los comicios efectuados, durante el año 2021 en Chile ratifican la valoración positiva, nacional y mundial, sobre la organización y ejecución de elecciones, más al considerar que se produjeron en tiempos de pandemia y en un contexto de una abierta pugnacidad política y sensibilidad social, lo cual deja de claro manifiesto la fortaleza institucional y la cultura ciudadana del país sureño.

\footnotetext{
52 Para ver posiciones al respecto, visitar https://www.latercera.com/politica/noticia/la-campana-sin-parisipdg-dice-que-derribamos-el-mito-de-que-el-viene-a-forrarse-y-expertos-reclaman-por-dano-a-fepublica/436UL3B575AOBJIMMYHSEGGS6E/
} 


\section{DERECHO ELECTORAL}

También ha quedado en evidencia, no obstante, que existen múltiples materias de carácter técnico electoral que deben ser abordadas, pues configuran áreas problemáticas o de mejoras, entre las cuales destacan: la formación y actualización del registro electoral, las inequidades aún existentes en el financiamiento de campañas electorales, la necesidad de regular los términos de los candidatos que van a la reelección, la rigurosidad en el manejo de las encuestas electorales, y la continuación y extensión de políticas afirmativas para las mujeres, independientes y pueblos originarios.

Dos temas no mencionados en el presente estudio pero que resultan transversales a todo el abordaje son, por una parte, la autonomía aún incompleta del Servicio Electoral (SERVEL), producto de la dualidad de instancias responsables de los procesos electorales ${ }^{53}$ y de la asunción de competencias de naturaleza electoral por parte de otros órganos del Estado como los poderes ejecutivo y legislativo. Casos como el de las reclamaciones por omisiones en el padrón electoral preliminar, función compartida por tribunales regionales electorales, el Tribunal Calificador de Elecciones (TRICEL) y el Servicio Electoral (SERVEL), y el de la postergación de la fecha de elecciones por motivo del COVID-19, acción que estuvo en manos del Ejecutivo nacional quien elevó la propuesta ante el Congreso, y de este que la aprobó finalmente, todo ello a pesar de que es el ente administrativo electoral a quien le corresponde formalmente organizar los procesos electorales $y$, además, por mera lógica, es el que posee la visión técnica más completa sobre el manejo de comicios y otro tipos de consultas; hacen temer que, a pesar de la autonomía formal del SERVEL, existe todavía un tutelaje del órgano electoral administrativo por parte de otras estructuras estatales ${ }^{54}$.

Por la otra parte, lo antes expuesto permite sugerir la necesidad de establecer legalmente la figura de la observación electoral en Chile ${ }^{55}$, nacional e internacional. Estos monitoreos podrían constituir una

\footnotetext{
53 En Chile existen dos organismos electorales supremos: el Servicio Electoral (SERVEL), de naturaleza administrativa y el Tribunal Calificador de Elecciones (TRICEL), de naturaleza jurisdiccional. Para ahondar en este punto se recomienda: Valdés, Leonardo y Ruiz, Fabián (2019) Los órganos encargados de la administración electoral en Nohlen, Dieter, Valdés, Leonardo y Zovatto, Daniel (2019), Derecho electoral Latinoamericano. FCE.

${ }^{54}$ Se recomienda la lectura de Hasbun, Cristóbal (2016) Tricel: Historia, legislación comparada y revisión de sus funciones. Recuperado de https://www.cepchile.cl/cep/site/docs/20160810/20160810100940/dpp_016_ago2016_chasbun.pdf

${ }^{55}$ Como complemento de este punto, se recomienda la lectura de Castellanos, Jesús (2020) ¿Es necesaria la observación electoral en Chile? https://demoamlat.com/wp-content/uploads/2021/06/REVISTA-DDAJUNIO2021-modificada.pdf
} 


\section{DERECHO EIECTORAL}

oportunidad de levantamiento de información técnica de manera rigurosa sobre el funcionamiento de los procesos electorales $y$, en consecuencia, la formulación de propuestas de reformas.

\section{REFERENCIAS BIBLIOGRÁFICAS}

Álvarez, A., Sajuria, G. y Ortiz, J. (2021). Lupa Electoral. Informe 2021. Recuperado de https://www.espaciopublico.cl/wpcontent/uploads/2021/08/INFORME-LUPA-ELECTORAL-2021.pdf

Bravo. J. (2021). Tres décadas de definiciones presidenciales. El Mercurio Infografía Digital. Recuperado de http://infografias.elmercurio.com/20171217-CPD-candidatospresidenciales/

Castellanos, J. (2019). Elecciones Chile 2020. Algunas propuestas de reforma. Revista DDA. (2), 23-27. Recuperado de https://www.demoamlat.com/wpcontent/uploads/2019/05/REVISTA-DDA-02-digital-comprimido.pdf

Chile (2012). Ley 20.568 que regula la inscripción automática, modifica el servicio electoral y moderniza el sistema de votaciones. Publicada en Diario Oficial el 31 de enero.

Chile (2017). Ley 20.640 sobre el marco regulatorio de las primarias en Chile. Publicado en el Diario Oficial el 6 de setiembre. Recuperado de https://www.diariooficial.interior.gob.cl/edicionelectronica/index.php?date $=0$ 6-09-2017\&edition $=41853$

Chile (2018). Ley 21.073 que regula la elección de gobernadores regionales y realiza adecuaciones a diversos cuerpos legales. Publicada en Diario Oficial n. ${ }^{\circ} 41.990$ del jueves 22 de febrero.

Chile (2019a). Acuerdo por la Paz Social y la Nueva Constitución. Recuperado de https://www.senado.cl/senado/site/docs/20191114/20191114134609/pdf_ac uerdo_por_la_paz_social_y_la_nueva_constitucion.pdf

Chile (2019b) Ley 21.200 que modifica el capítulo XV de la Constitución Política de la República. Publicada en Diario Oficial n. ${ }^{\circ} 42.536$ del martes 24 de diciembre.

Chile (2020). Ley 21.238 sobre el límite a la reelección de autoridades. Publicado en el Diario Oficial 42.700 del miércoles 8 de julio. Recuperado de https://www.bcn.cl/leychile/navegar?idNorma $=1147301$ 


\section{DERECHO EIECTORAL}

Chile (2021). Ley 21.324 que posterga las próximas elecciones municipales, de gobernadores regionales y de convencionales constituyentes por motivo del covid-19. Publicada en Diario Oficial n. ${ }^{\circ} 42.923$ del miércoles 7 de abril.

Consejo Asesor Presidencial contra los Conflictos de Interés, el Tráfico de Influencias y la Corrupción (2015). Informe final. Recuperado de http://consejoanticorrupcion.cl/wp-content/uploads/2015/06/2015.06.05consejo_anticorrupcion.pdf, en su informe de 2015

Consejo Nacional de Televisión de Chile (2021). Resolución 512 que establece reglamento para la franja electoral de la elección primaria presidencial de 18 de julio de 2021. Publicada en el Diario Oficial el 9 de junio. Recuperado de https://www.diariooficial.interior.gob.cl/edicionelectronica/index.php?date $=0$ 9-06-2021\&edition $=42974$

Contraloría General de la República de Chile (2016). Estudio sobre gastos en publicidad y difusión en el sector municipal 2006. Recuperado de http://sistemas.contraloria.cl/portalweb/documents/451102/2132612/Estudi o2006-2016+\%281\%29.pdf/17c865bc-4ada-9f46-b8d6-d8b1c3e7cc0d

Contraloría General de la República de Chile (2020). Dictamen E5031 del 10 de noviembre. Recuperado de https://www.contraloria.cl/pdfbuscador/dictamenes/E50319N20/html

Corporación Latinobarómetro (2021) Informe Chile 2020. Recuperado de https://www.latinobarometro.org/latdocs/INFORME_LATINOBAROMETRO_CH ILE_1995_2020.pdf

Debates y nudos críticos en la convención constitucional chilena (2021). Agenda Pública. Recuperado de https://agendapublica.es/debates-y-nudos-criticosen-la-convencion-constitucional-chilena/

Decide Chile (2021). Elección 2021. Constituyentes. Recuperado de https://2021.decidechile.cl/\#/ev/2021/ct/2021.N/

Democracy Index (2020). In sickness and in health? The Economist Recuperado de https://pages.eiu.com/rs/753-RIQ-438/images/democracy-index2020.pdf?mkt_tok=NzUzLVJJUS00MzgAAAGAgCs8ZYFWrAyqekRXTRfNSY_kF $85-$

6wbuQfVmBL83IpPmXgQWdCm7RMXtxehofiWjHpe5m5LpEyXCS1EyNZONFnl KjOmOosOngjSMJ6uOUauOoA

Electoral Integrity Report (2019). Recuperado de https://www.electoralintegrityproject.com/the-year-in-elections-2019 


\section{DERECHO ELECTORAL}

Engel, E. y otros (2018). Análisis de implementación de las nuevas reglas de financiamiento a la política y campaña electoral. Lupa Electoral 2016 y 2017. Recuperado de https://espaciopublico.cl/wpcontent/uploads/2021/05/Informe-Lupa-electoral.pdf

Figueroa, P. (Ago.-Set., 2021). La Convención Constitucional de Chile: Un desafío de inclusión y legitimidad democrática. Revista Ideele, (299), sin paginación. Recuperado de https://www.revistaideele.com/2021/09/03/la-convencionconstitucional-de-chile-un-desafio-de-inclusion-y-legitimidad-democratica/

Freedom in the World (2021). Recuperado de https://freedomhouse.org/country/chile/freedom-world/2021

Índice de Estado de Derecho (2020). Recuperado de https://worldjusticeproject.org/sites/default/files/documents/WJP-GlobalROLI-Spanish.pdf

Programa de las Naciones Unidas para el Desarrollo (Chile) (2018). Representación política de mujeres en el Poder Legislativo. Análisis de la aplicación de cuotas de género en las elecciones parlamentarias 2017. Chile: PNUD. Recuperado de

https://www.cl.undp.org/content/chile/es/home/library/democratic_governan ce/representacion-politica-de-mujeres-en-el-poder-legislativo.html

Ríos, M. (2021). Participación electoral y voto obligatorio. Chile: PNUD. Recuperado de https://www.cl.undp.org/content/dam/chile/docs/CL\%20Reimplementaci\%C 3\%B3n\%20voto\%20obligatorio\%20Analisis\%20PNUD\%2031\%20mayo\%202 021.pdf

Servicio Electoral de Chile (2020). Manual sobre publicidad y propaganda electoral. Elecciones constituyentes, gobernadores, alcaldes y concejales, 2021. Chile: Servicio Electoral de Chile. Recuperado de https://www.servel.cl/wpcontent/uploads/2021/01/MANUAL_PROPAGANDA_ABRIL_2021_.pdf

Servicio Electoral de Chile (2021). Manual sobre publicidad y propaganda electoral. Chile: Servicio Electoral de Chile. Recuperado de manual_propaganda_elecciones_nov.pdf (windows.net)

Wilson, J. (31 de mayo de 2021). Los 3 factores que incidieron en la baja participación de las elecciones de mayo, según el Servel. Ex-ante. Recuperado de https://www.ex-ante.cl/https-www-ex-ante-cl-los-3-factoresque-incidieron-en-la-baja-participacion-de-las-elecciones-de-mayo-segun-elservel/ 